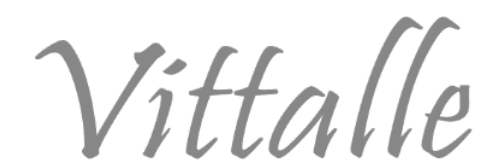

\title{
Fatores relacionados à baixa adesão ao tratamento de hipertensos e diabéticos
}

\author{
Taline Masson $^{\mathrm{a}}$, Fabiana Meneghetti Dallacosta ${ }^{\mathrm{b}^{*}}$

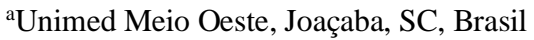 \\ ${ }^{b}$ Universidade do Oeste de Santa Catarina, Joaçaba, SC, Brasil
}

\section{Histórico do Artigo \\ Recebido em: \\ 01/10/2021 \\ Aceito em: \\ $12 / 11 / 2021$}

Palavras-chave: Hipertensão; Diabetes Mellitus; estilo de vida

Keywords:

Hypertension; Diabetes Mellitus; lifestyle

\begin{abstract}
RESUMO
A adesão ao tratamento é fundamental para o sucesso da terapêutica nos portadores de doenças crônicas. O objetivo do estudo foi avaliar os fatores relacionados a baixa adesão ao tratamento de hipertensos e diabéticos, relacionando com o estilo de vida. Foi realizado um estudo transversal, com hipertensos e diabéticos de um município de Santa Catarina. Para análise da adesão ao tratamento foi usado o Brief Medication Questionnaire, e para o estilo de vida foi usado o questionário Fantástico.Participaram 308 hipertensos e diabéticos, média de idade $65,4 \pm 10,5$ anos, $96,1 \%$ hipertensos e $27,3 \%$ diabéticos. Aderentes ao tratamento totalizaram $19,8 \%, 28,2 \%$ são prováveis aderentes, $23,4 \%$ tem provável baixa adesão e $28,6 \%$ possuem baixa adesão. Quanto ao estilo de vida, 18,2\% mantém um estilo de vida "Excelente", 57,7\% "Muito Bom", 20,8\% "Bom" e, 2,6\% "Regular". O Índice de Massa Corpórea (IMC) das pessoas acima de 60 anos foi menor que dos mais jovens $(p=0,00)$ e o IMC dos diabéticos foi maior que dos hipertensos $(\mathrm{p}=0,00)$. Aqueles que relataram que consumiam alimentos saudáveis, são os que têm IMC menor $(\mathrm{p}=0,02)$. Os usuários que realizam atividade física frequentemente possuem estilo de vida melhor quando comparados com quem pratica atividade física eventualmente $(\mathrm{p}=0,00)$. A adesão ainda é um desafio para os profissionais que atuam com hipertensos e diabéticos, e os cuidados relativos à prática de exercícios e controle do peso devem ser incentivados como estratégia para diminuir o risco de co-morbidades e complicações.
\end{abstract}

Factors related to low adherence to treatment for hypertensive and diabetic patients

\begin{abstract}
Adherence to treatment is essential for successful therapy in patients with chronic diseases, relating to lifestyle. The aim of the study was to evaluate the factors associated to poor adherence to treatment of hypertensives and diabetics. A cross-sectional study was conducted with hypertensive and diabetic patients from a city in Santa Catarina. To analyze the adherence to treatment was used the Brief Medication Questionnaire, and for the lifestyle the Fantastic Questionnaire. Participated 308 hypertensives and diabetics, average age $65,4 \pm 10,5$ years, $96,1 \%$ hypertensives and $27,3 \%$ diabetics. Adherents to treatment was $19.8 \%, 28.2 \%$ were likely adherents, $23.4 \%$ probably low adherents and $28.6 \%$ with low adherence. As for lifestyle, $18.2 \%$ maintains an "Excellent" lifestyle, 57.7\% "Very Good", 20.8\% "Good" and 2.6\% "Regular". The Body Mass Index (BMI) of people above 60 years was lower than youngers $(p=0.00)$ and the BMI of diabetics was higher than hypertensives $(\mathrm{p}=0.00)$. Those who reported eating healthy foods were the ones with the lowest BMI $(\mathrm{p}=0.02)$. Users who perform physical activity often, have a better lifestyle when compared with those who practice physical activity eventually $(\mathrm{p}=0.00)$. Adherence is still a challenge for professionals working with hypertensive and diabetic patients, and care related to exercise and weight control should be encouraged as a strategy to reduce the risk of comorbidities and complications.
\end{abstract}

\section{Introdução}

Diabetes mellitus (DM) e hipertensão arterial sistêmica (HAS) são doenças crônicas não transmissíveis (DCNT), consideradas problemas de saúde pública de grande magnitude, sendo que o Brasil tem o maior número de pessoas com DM entre os países da América

\footnotetext{
*Autor correspondente: fabiana.dallacosta@unoesc.edu.br_(Dallacosta F.M.)
} 
do Sul e Central (1). A hipertensão é a doença crônica de maior prevalência entre os países desenvolvidos e em desenvolvimento, acometendo $30 \%$ da população brasileira com 18 anos ou mais (2).

Diabetes e hipertensão constituem a principal causa de morbimortalidade no Brasil e são responsáveis por importante impacto social e econômico, e as complicações decorrentes destas patologias são agravadas pela dificuldade dos pacientes em aderir ao tratamento, que envolve o uso constante de medicamentos, mudanças no perfil alimentar e no estilo de vida (3).

Um dos desafios para a equipe interdisciplinar é melhorar a adesão e a qualidade de vida de hipertensos e diabéticos, a fim de evitar gastos em saúde e reduzir a mortalidade e complicações relacionadas a doença. A adesão ao tratamento influencia na sobrevida e na qualidade de vida dos pacientes, e deve ser constantemente analisada e incentivada, para que os portadores de doenças crônicas façam uso correto das medicações, e reportem à equipe os motivos da baixa adesão ou as dificuldades encontradas no uso destas (4).

O objetivo deste estudo foi avaliar os fatores relacionados a baixa adesão ao tratamento de hipertensos e diabéticos, relacionando com o estilo de vida.

\section{Materiais e Métodos}

Trata-se de um estudo transversal, realizado em um munícipio de Santa Catarina, que possui 328 hipertensos e/ou diabéticos cadastrados na Unidade Básica de Saúde (UBS). Como critérios de inclusão foram considerados estar vinculado a UBS, ter idade acima de 18 anos e possuir diagnóstico de hipertensão ou diabetes. Excluíram-se aqueles pacientes que não aceitaram participar do estudo ou não conseguiram responder a todos instrumentos desta pesquisa. Os dados foram coletados diretamente pelas pesquisadoras, mediante visita domiciliar aos usuários cadastrados na unidade de saúde.

Para análise da adesão ao tratamento foi usado o Brief Medication Questionnaire, que analisa aspectos relacionados ao uso de medicação oral quanto à três aspectos: Crenças, Regime e Recordação. Nenhuma resposta positiva indica adesão ao tratamento, uma resposta positiva sugere provável adesão ao tratamento, duas respostas e três ou mais positivas indicam, respectivamente, provável baixa adesão e baixa adesão ao tratamento (5).

Para análise dos hábitos de vida foi utilizado o questionário "Estilo de vida Fantástico", que considera o comportamento dos indivíduos no último mês e cujos resultados permitem determinar a associação entre o estilo de vida e a saúde. O instrumento possui 25 questões divididas em nove domínios que são: 1) família e amigos; 2) atividade física; 3) nutrição; 4) cigarro e drogas; 5) álcool; 6) sono, cinto de segurança, estresse e sexo seguro; 7) tipo de comportamento; 8) introspecção; 9) trabalho. As questões estão dispostas na forma de escala Likert, 23 possuem cinco alternativas de resposta e duas são dicotômicas. A codificação das questões é realizada por pontos: zero para a primeira coluna, 1 para a segunda coluna, 2 para a terceira coluna, 3 para a quarta coluna e 4 para a quinta coluna. As questões que só possuem duas alternativas pontuam: zero para a primeira coluna e 4 pontos para a última coluna. A soma de todos os pontos permite chegar a um escore total que classifica os indivíduos em cinco categorias que são: "Excelente" (85 a 100 pontos), "Muito bom" (70 a 84 pontos), "Bom" (55 a 69 pontos), "Regular" (35 a 54 pontos) e "Necessita melhorar" (0 a 34 pontos).

A origem da palavra "fantástico" vem do acrônimo FANTASTIC que representa as letras dos nomes dos nove domínios (na língua inglesa) em que estão distribuídas as 25 questões ou itens: $\mathrm{F}=$ Family and friends (família e amigos); $\mathrm{A}=$ Activity (atividade física); $\mathrm{N}=$ Nutrition (nutrição); $\mathrm{T}=$ Tobacco \& toxics(cigarro e drogas); $\mathrm{A}=$ Alcoho $l($ álcool); $\mathrm{S}=$ Sleep, seatbelts, stress, safe sex (sono, cinto de segurança, estresse, sexo 
seguro); $\mathrm{T}=$ Type of behavior (tipo de comportamento; padrão de comportamento A ou B);I= Insight (introspecção);C= Career (trabalho; satisfação com a profissão).

Os dados quantitativos foram descritos por média e desvio-padrão e os categóricos por contagens e percentuais. A comparação de variáveis quantitativas entre grupos foi realizada pelo teste $t$ Student, a associação de variáveis quantitativas entre si foi realizada utilizando-se o coeficiente de correlação de Pearson. Os cruzamentos de dados categóricos foram realizados pelo teste de Qui-quadrado e Teste Exato de Fisher. O nível de significância adotado foi de 95\%. Foi utilizado o programa estatístico SPSS 21.0.

O projeto foi aprovado pelo Comitê de Ética em Pesquisa da Universidade do Oeste de Santa Catarina (Unoesc), sob o parecer n. 2.578.699 e todos preceitos éticos foram seguidos, conforme Resolução Conselho Nacional de Saúde nº 466, de 12 de dezembro de 2012. Todos participantes assinaram o Termo de Consentimento Livre e Esclarecido.

\section{Resultados}

Dos 328 indivíduos cadastrados, 20 foram excluídos, totalizando 308 participantes. A idade média foi de 65,4 $\pm 10,5$ anos, $75,3 \%$ com menos de oito anos de estudo, $72 \%$ tem renda inferior a dois salários mínimos. As demais características sócio demográficas estão descritas na Tabela 1.

A avaliação da adesão ao tratamento medicamentoso revelou que $19,8 \%$ dos hipertensos e diabéticos são aderentes, $28,2 \%$ são prováveis aderentes, $23,4 \%$ classificaram-se como provável baixa adesão e 28,6\% possuem baixa adesão.

Em relação ao estilo de vida, 18,2\% mantém um estilo de vida "Excelente", 57,7\% "Muito Bom", 20,8\% "Bom", 2,6\% "Regular" e 0,7\% possuem um estilo de vida que "precisa melhorar". O estilo de vida não apresentou relação com a adesão ao tratamento $(\mathrm{p}=0,56)$.

Tabela 1 - Características sóciodemográficas dos hipertensos e diabéticos. N= 308

\begin{tabular}{lcc}
\hline Variável & $\mathbf{N}$ & $\%$ \\
\hline Sexo & 189 & 61,4 \\
Feminino & 119 & 38,6 \\
Masculino & 296 & 72,7 \\
Hipertensos & 84 & 27,3 \\
Diabéticos & & \\
Etnia & 275 & 89,3 \\
Brancos & 27 & 8,8 \\
Negros & 6 & 1,9 \\
Outros & & \\
Situação conjugal & 207 & 67,2 \\
Com companheiro & 101 & 32,8 \\
Sem companheiro & & \\
Tabagismo & 30 & 9,7 \\
Fumante & 65 & 21,1 \\
Ex-fumante & 213 & 69,2 \\
Nunca fumou & & \\
\hline
\end{tabular}

A média do questionário Fantástico foi de $75,7 \pm 10,0$ pontos, que se enquadra na categoria de 70 a 84 pontos, ou seja, qualidade de vida "Muito Boa". A pontuação mínima obtida foi de 40 e a máxima de 98 pontos. Não houve diferença estatística na média de pontuação dos aderentes $(75,9 \pm 10,5$ pontos $)$ comparado aos não aderentes $(75,6 \pm 9,5$ pontos) $(\mathrm{p}=0,77)$.

Analisando outros aspectos do questionário Fantástico, observamos que 87,4\% dos entrevistados informam que possuem quase sempre alguém para conversar, $79,8 \%$ dão e recebem afeto, 53,4\% referiram que pensam de forma positiva e otimista, 44,6\% quase 
sempre dormem bem e sentem-se descansados, 76,9\% estão quase sempre satisfeitos com seu trabalho ou função.

Na categoria de introspecção, 46,9\% sentem-se tensos e desapontados e 41,4\% sentemse tensos e deprimidos algumas vezes. Quanto ao tipo de comportamento, 39,1\% dos usuários refere sentir raiva e hostilidade algumas vezes e 48,9\% quase nunca aparentam estar com pressa.

O consumo de álcool foi referido por 35\%, e desses, 93,8\% consomem de zero a sete doses de álcool na semana, 93,8\% relataram não dirigir após beber, e 92,2\% nunca bebe mais do que quatro doses em uma ocasião. Dentre os entrevistados, 99,3\% nunca usaram drogas, e $80,5 \%$ nunca abusaram/exageraram no consumo de remédios.

Sobre os hábitos alimentares, 63,2\% referiram que consomem uma dieta balanceada quase sempre, $36,5 \%$ frequentemente comem em excesso um item (sal/açúcar/gordura/salgadinhos) e 72,6\% ingerem bebidas que contém cafeína uma a duas vezes por dia. Em relação aos parâmetros biométricos, observou-se que a média do Índice de Massa Corporal (IMC) foi de $28,4 \mathrm{~kg} / \mathrm{m}^{2}$ e $53,2 \%$ estão com mais de $8 \mathrm{~kg}$ do peso considerado saudável/ideal

Quanto à quantidade de medicamentos, 56,8\% recebe um esquema de múltiplas doses ( 2 ou mais vezes/dia), 28,6\% dos usuários relataram que é muito difícil ler o que está escrito na embalagem, $11,7 \%$ tem muita dificuldade para conseguir o medicamento, $53,2 \%$ falhou em listar (espontaneamente) os medicamentos que usa, 18,2\% relatou que já reduziu ou omitiu doses de algum medicamento e $24,4 \%$ referiram que às vezes esquecem de tomar os comprimidos. Analisando a relação da falha de dias ou doses com o estilo de vida, usuários com ausência de falha de dias possuem um estilo de vida melhor do que os que relataram falhas $(\mathrm{p}=0,01)$.

Analisando a Tabela 2 observa-se que aqueles acima de 60 anos foram mais aderentes $(\mathrm{p}=0,00)$ e os que têm melhor estilo de vida $(\mathrm{p}=0,05)$. Os diabéticos foram menos aderentes quando comparados aos hipertensos $(\mathrm{p}=0,01)$ e verificou-se que quem usa múltiplas medicações possui menor adesão $(\mathrm{p}=0,00)$.

Tabela 2 - Relação da adesão ao tratamento com variáveis sociodemográficas e de saúde.

\begin{tabular}{|c|c|c|c|c|}
\hline Variáveis & $\begin{array}{l}\text { Total } \\
\text { n }(\%)\end{array}$ & $\begin{array}{c}\text { Aderentes } \\
\text { n (\%) }\end{array}$ & $\begin{array}{c}\text { Não Aderentes } \\
\text { n (\%) }\end{array}$ & $\mathbf{p}^{*}$ \\
\hline \multicolumn{5}{|l|}{ Sexo } \\
\hline Masculino & $119(38,6)$ & $56(37,8)$ & $63(33,1)$ & 0,78 \\
\hline Feminino & $189(61,3)$ & $92(62,2)$ & $97(66,9)$ & \\
\hline \multicolumn{5}{|l|}{ Faixa etária } \\
\hline$>60$ anos & $225(73)$ & $97(65,5)$ & $128(80,0)$ & 0,00 \\
\hline$<59$ anos & $83(27)$ & $51(34,5)$ & $32(20,0)$ & \\
\hline \multicolumn{5}{|l|}{ Ativos fisicamente } \\
\hline Sim & $129(42)$ & $65(43,9)$ & $64(40,0)$ & 0,48 \\
\hline Não & $179(58)$ & $83(59,1)$ & $96(60,0)$ & \\
\hline \multicolumn{5}{|c|}{ Múltiplas doses medicamento } \\
\hline Sim & $175(56,8)$ & $60(40,5)$ & $115(71,8)$ & 0,00 \\
\hline Não & $133(43,2)$ & $88(59,5)$ & $45(28,2)$ & \\
\hline \multicolumn{5}{|l|}{ Omitiram medicacões } \\
\hline Sim & $56(18)$ & $01(0,7)$ & $55(34,3)$ & 0,00 \\
\hline Não & $252(82)$ & $147(99,3)$ & $105(65,7)$ & \\
\hline \multicolumn{5}{|l|}{ Diabéticos } \\
\hline Sim & $84(27,2)$ & $31(20,9)$ & $53(33,1)$ & 0,01 \\
\hline Não & $224(72,7)$ & $117(79,1)$ & $107(66,9)$ & \\
\hline \multicolumn{5}{|l|}{ Estilo de vida } \\
\hline Excelente & $56(18,1)$ & $28(50)$ & $28(50)$ & \\
\hline Muito bom & $177(57,4)$ & $81(45,7)$ & $96(44,3)$ & \\
\hline Bom & $64(20,7)$ & $33(51,5)$ & $31(48,5)$ & 0,48 \\
\hline Regular & $08(2,5)$ & $04(50)$ & $04(50)$ & \\
\hline Precisa melhorar & $02(0,6)$ & $02(100)$ & 00 & \\
\hline Total & $308(100)$ & $148(48,0 \%)$ & $160(52,0 \%)$ & \\
\hline
\end{tabular}


Constatou-se que o IMC das pessoas acima de 60 anos é menor que dos mais jovens ( $\mathrm{p}=$ 0,00), que o IMC e os escores referente ao Estilo de Vida das pessoas com menos de 39 anos foram os piores $(\mathrm{p}=0,01)$. O IMC dos diabéticos foi maior que dos hipertensos $(\mathrm{p}=$ $0,00)$ e aqueles que relataram que consumiam alimentos saudáveis, são os que têm IMC menor $(\mathrm{p}=0,02)$.

Os sedentários correspondem a 57,8\%, mas $63 \%$ informaram que são vigorosamente ativos pelo menos 30 minutos por dia, mas apenas uma a duas vezes por semana; e 34,4\% são moderadamente ativos por cinco ou mais vezes por semana, realizando caminhada, trabalho de casa e jardinagem.

Aqueles que realizam atividade física frequentemente, possuem estilo de vida melhor quando comparados com quem pratica atividade física eventualmente $(\mathrm{p}=0,00)$.

\section{Discussão}

Observou-se baixa prevalência de aderentes ao tratamento. O baixo nível de instrução é comum nas pesquisas realizadas com esta população, fator que pode limitar o acesso às informações, diminuindo a compreensão das orientações recebidas pelos profissionais (6).

Não houve diferença na adesão considerando sexo e estado civil, ainda que a presença constante de um familiar possa incentivar o tratamento adequado, atendimento das necessidades e cuidados diários (6). Outros estudos semelhantes têm mostrado resultados que corroboram com esses achados, onde sexo e estado civil não interferem na adesão ao tratamento $(7,8)$.

Os mais velhos foram mais aderentes, mas de modo geral a baixa adesão medicamentosa é comum entre os idosos, relacionando-se com a complexidade dos esquemas medicamentosos, falta de entendimento, diminuição da acuidade visual e o esquecimento (9). Estudo realizado com portadores de doença renal mostrou que a adesão ao tratamento foi pior em pessoas acima de 61 anos, incluindo nessa faixa etária piores índices de letramento em saúde, que contribui para uma pior aderência à terapêutica (10). Um fator decisivo para aumentar adesão é a confiança do paciente na equipe de saúde, podendo ser estimulada por meio do tempo dispensado nas consultas, atendimento acolhedor e linguagem adequada ao paciente (11). No tratamento da HAS e DM, a primeira alternativa terapêutica deve ser a mudança do estilo de vida. A maior adesão à mudança do estilo de vida ocorre quando mais de um profissional orienta, diferentes tipos de abordagens são importantes para que o paciente compreenda a relevância da doença (12).

A abordagem sobre o estilo de vida não é um processo fácil e linear, faz-se necessário a motivação e desejo do paciente, avaliar a oferta de serviços de saúde à população, condições socioeconômicas e desigualdades sociais (13). Além disso, deve-se considerar que os hábitos de vida são comportamentos sociais e culturais construídos ao longo da vida, marcados por simbologias e tabus, de difícil modificação, especialmente quando se trata de idosos (14). O melhor método são as orientações preventivas, como a boa nutrição, controle do peso e atividades físicas que devem durar por toda uma vida (15). Dentro desse contexto, faz-se necessário compreender a dimensão psíquica do cuidado, pois o tratamento não engloba somente a administração de medicamentos, devendo abranger também os aspectos emocionais (13).

Ao serem questionados nos domínios de cigarro e álcool, verificou-se pequena prevalência de fumantes. A diminuição do número de fumantes pode estar relacionada com o acesso ao conhecimento sobre prejuízos do tabaco à saúde, associado às ações desenvolvidas pelo Governo Federal, como por exemplo, o aumento dos impostos e do 
preço do tabaco (16). De acordo com Teixeira et al. (13), comportamentos aditivos, como o etilismo e o tabagismo, fizeram ou fazem parte dos hábitos de vida, forma que o indivíduo encontra para aliviar angústias e ter gratificação emocional.

Aqueles pacientes que usam múltiplas doses diárias e que relataram já ter feito omissão de doses prescritas foram menos aderentes. O maior número de medicamentos prescritos está associado a não-adesão, pois os esquemas terapêuticos geralmente são mais complicados e exigem maior dedicação por parte do paciente (11). Os resultados insatisfatórios relatados mostram, em conjunto, que os pacientes não entendem e tem dificuldade para compreender quais medicamentos deveriam tomar, no horário e forma prescritos, por mais que tenham interesse em modificar seu estilo de vida e seguir a prescrição médica e orientação da equipe (16).

O uso de múltiplas doses é um fator que contribui para pior adesão à terapêutica prescrita, o que já foi demonstrado em outros estudos semelhantes com hipertensos e diabéticos, devendo, portanto, ser um fator de atenção para a equipe de saúde, que precisa monitorar e orientar constantemente esses pacientes para melhores resultados $(7,8)$.

Ressalta-se a importância dos grupos Hiperdia no atendimento e condução do tratamento dos hipertensos e diabéticos, atuando de forma interdisciplinar. O programa é importante ao promover adesão ao tratamento farmacológico e não farmacológico, assim como na redução de agravos, ao atuar na prevenção e controle da HAS e DM, por meio do desenvolvimento de um vínculo entre os pacientes e a equipe de saúde.

\section{Considerações finais}

A adesão ao tratamento entre os hipertensos e diabéticos foi baixa, e os menos aderentes foram os mais velhos, que usam múltiplas doses de medicação e que já omitiram doses do seu tratamento. $\mathrm{O}$ estilo de vida foi considerado excelente ou muito bom para a maioria dos participantes. Ter alimentação saudável e praticar atividades físicas obteve relação com o estilo de vida mais saudável e com menor peso e IMC mais baixo, o que deve ser continuamente incentivado nesta população.

Os achados obtidos podem ser utilizados orientar as atividades desenvolvidas na atenção primária, facilitando o trabalho dos profissionais de saúde no desenvolvimento de uma abordagem diferenciada junto aos pacientes vulneráveis. Dessa forma, estratégias educacionais compatíveis com a realidade, serão capazes de contribuir para a adesão ao tratamento e qualidade de vida e, consequentemente, diminuir os riscos de complicações e comorbidades.

Este estudo teve como limitação o seu desenho transversal, que analisou um período específico de tempo, sendo recomendado novos estudos longitudinais para avaliar o impacto da baixa adesão ao longo da vida.

\section{Referências}

1. International Diabetes Federation. IDF Diabetes Atlas 8 th-edition: 2017. p.77. Disponível em: https://www.idf.org/e-library/epidemiology-research/diabetes-atlas/134-idf-diabetes-atlas-8thedition.html.

2. Malachias MVB et al. $7^{\text {a }}$ Diretriz Brasileira de Hipertensão Arterial. Arq Bras Cardiol 2016; 107 (3): 1-83. Disponível em: http://publicacoes.cardiol.br/2014/diretrizes/2016/05_HIPERTENSAO_ARTERIAL.pdf.

3. Faria HTG, Santos MA, Arrelias CCA, Rodrigues FFL, Gonela JT, Teixeira, CRS, Zanetti ML. Adesão ao tratamento em diabetes mellitus em unidades da Estratégia Saúde da Família. Rev Esc Enferm USP. 2014; 48(2): 257-63.

4. Mantovani MF, Mattei ÂT, Arthur JP, Ulbrich EM, Moreira RC. Utilização do brief medication questionnaire na adesão medicamentosa de hipertensos. Rev enferm UFPE on line 2015; 9(1): 84-90. 
5. Silva AL, Fonseca GSS, Rossi VEC. Adesão de pessoas com diabetes mellitus tipo 2 ao tratamento medicamentoso. Rev Bras Enferm. 2014; 67(2): 21 - 26.

6. Gellad WF, Grenard JL, Marcum ZA. A systematic review of barriers to medication adherence in the elderly: looking beyond cost and regimen complexity. Am J Geriatr Pharmacother 2011; 9(1):11-23.

7. Dallacosta FM, Restelatto MTR, Turra L. Adesão ao tratamento e hábitos de vida de hipertensos. Rev Fun Care Online. 2019; 11(1): 113-117.

8. Zancanaro S., Rossoni C., Dallacosta FM. Adherence to treatment for diabetes and relation with kidney function and lifestyle. Int J of Develop Research. 2018; 8(12): 24806-24809.

9. Leite SN, Vasconcellos MPC. Adesão à terapêutica medicamentosa: elementos para discussão de conceitos e pressupostos adotados na literatura. Ciênc Saúde Col. 2003; 8(3): 775-782.

10. Santos DB, Rossoni C, Dallacosta FM. Health Literacy and Relation to Adherence to Pharmacologic Treatment of Patients in Hemodialysis. Int J of Advanced Engineering Research and Science. 2021; 8(8): 454-459.

11. Rohrbacher I, Correa CJS, Schmitz GLP, Romulo MCB, Gonçalves PCZ. Orientações de mudança de estilo de vida em pacientes hipertensos. Rev AMRIGS. 2014; 58(1): 49-53.

12. Teixeira ER, Lamas AR, Costa e Silva J, Matos RM. O estilo de vida do cliente com hipertensão arterial e o cuidado com a saúde. Esc. Anna Nery R Enferm. 2016; 10(3): 378 - 84.

13. Borba ACOT, Marques APO, Ramos VP, Leal MCC, Arruda IKG, Ramos RSPS. Fatores associados à adesão terapêutica em idosos diabéticos assistidos na atenção primária de saúde. Ciênc saúde coletiva. 2018; 23 (3); 53-961.

14. Porto EF, Kumpel C, Castro AAM, Oliveira IM, Alfieri FM. Como o estilo de vida tem sido avaliado: revisão sistemática. Acta Fisiátrica. 2015; 22 (4): 199-205.

15. Figueiredo VC, Turci SB, Camacho LAB. Controle do tabaco no Brasil: avanços e desafios de uma política bem-sucedida. Cad. Saúde Pública. 2017; 33 (suppl. 3), e00104917.

16. Abulquerque GSC, Nascimento B, Gracia DFK, Preisler L, Perna PO, Souza e Silva MJ. Adesão de hipertensos e diabéticos analfabetos ao uso de medicamento a partir da prescrição pictográfica. Trab Educ Saúde. 2016; 14 (2), 611- 624. 